\title{
Nanotechnology applied to drug delivery - formulation, development and characterization studies
}

\author{
A nanotecnologia aplicada à veiculação de fármacos: estudos de desenvolvimento da \\ formulação e caracterização
}

\author{
Catarina Pinto Reis ${ }^{1}$, Pedro C. Pinto ${ }^{2}$, Ana Gomes ${ }^{1} \&$ Sara Candeias ${ }^{1}$ \\ CBIOS - Research Center for Health Science and Technologies, Universidade Lusófona, Campo Grande, 376, \\ 1749-024, Lisboa, Portugal \\ ${ }^{1}$ LNBN - Laboratory of Nanoscience and Biomedical Nanotechnology \\ ${ }^{2}$ UDE - Experimental Dermatology Unit (UDE) \\ Email: catarina.reis@ulusofona.pt
}

\begin{abstract}
Azelaic acid shows bacteriostatical activity against many microorganism species and is commonly used in the treatment of acne. However, some technical issues and a reduced patient compliance have been associated with its topical application forms. Thus, nanotechnology may represent an innovative strategy that might help to overcome these problems. The objective of this study was to develop and to characterize PLGA nanoparticles containing azelaic acid. Nanoparticles were produced by a modified spontaneous emulsification/solvent diffusion method and then included into a gel of Carbopol 940. Several parameters were characterized such as zeta potential, particle size and encapsulation efficiency. Particle mean size was $378.63 \mathrm{~nm}$ (with PI around 0.09) and zeta potential was -7.82 $\mathrm{mV}$. The encapsulation efficiency of azelaic acid was $76 \pm 3.81 \%$. Consequently, these PLGA nanoparticles can be considered a useful tool for azelaic acid delivery.
\end{abstract}

Keywords: Azelaic Acid; PLGA; Nanoparticles; Formulation development and characterization

\begin{abstract}
Resumo
O ácido azelaico é um fármaco com actividade bacteriostática para muitos microorganismos sendo por isso frequentemente aplicado no tratamento do acne. Porém, às formulações tópicas deste fármaco estão geralmente associados alguns efeitos adversos e fracas adesões à terapêutica. Assim, a nanotecnologia pode ser aqui considerada como uma estratégia inovadora para ultrapassar os obstáculos anteriores. O objectivo deste estudo centrou-se no desenvolvimento e caracterização de nanopartículas de PLGA contendo o ácido azelaico. As nanopartículas foram produzidas através do método modificado de emulsificação/difusão do solvente e posteriormente incluídas num gel de Carbopol 940. Foram caracterizados vários parâmetros da formulação tais como potencial zeta, tamanho da partícula e eficiência de encapsulação. O tamanho médio das partículas foi de 378,63 nm (com I.P. cerca de 0,09) e o potencial zeta foi de $-7,82 \mathrm{mV}$. A eficiência de encapsulação do ácido azelaico foi de $76 \pm 3,81 \%$. Consequentemente, estas nanopartículas poderão ser consideradas ferramentas úteis para a veiculação do ácido azelaico.
\end{abstract}

Palavras-chave: Ácido azelaico, PLGA; Nanopartículas; Desenvolvimento da formulação e caracterização. 


\section{Introduction}

Polymeric nanoparticles show great promise in the treatment of a wide range of diseases due to the flexibility in which their structures can be modified. These delivery systems have been proven to enhance the effectiveness of many drugs ${ }^{[1-4]}$ and have shown some benefits in skin permeation ${ }^{[3-4]}$. The skin, the largest and heaviest human organ, provides a barrier to exogenous chemicals such as drugs. The outermost layer of the skin is the stratum corneum. There are two different ways for drugs to permeate the stratum corneum - through the transepidermal route or via pores. The pores' route includes skin appendages ${ }^{[5]}$. Pilosebaceous units are composed of hair follicles and sebaceous glands ${ }^{[6]}$. A problem commonly associated with skin topical drug formulations is the lack of permeation mainly due to reduced contact time, solubilization problems and drug stability. Polymeric nanoparticles are expected to increase the drug's stability, the residence time of the formulation, and consequently, to increase the drug absorption probability. A previously published study ${ }^{[7]}$ has shown that the particle permeation through hair follicles was associated with the pores via absorption.

In this study, azelaic acid (AzA) was used as a model drug. AzA possesses bacteriostatical, antiinflammatory and antikeratinizing properties, and is commonly applied in acne treatment ${ }^{[8-9]}$. AzA has shown no bacterial resistance even after long exposure and has also a favorable tolerability profile $\left.{ }^{[8,10}\right]$. However, this drug may require several daily applications and, furthermore, solubilization is generally the rate-limiting step in percutaneous absorption of $\mathrm{AzA}^{[11]}$. The objective of this study was to develop a delivery system based on PLGA nanoparticles containing azelaic acid. PLGA (PolyDL-Lactide/Glycolide copolymer), a biodegradable polymer, has been broadly used for controlled release formulations and displays a very favourable degradation profile ${ }^{[12]}$. In the present study, several parameters were evaluated such as particle size, zeta potential and encapsulation efficiency.

\section{Introdução}

As nanopartículas poliméricas surgem como uma alternativa promissora no tratamento de muitas doenças principalmente devido à flexibilidade de alteração das suas estruturas. Estes sistemas de veiculação já demonstraram um aumento de eficácia de muitos fármacos ${ }^{[1-3]}$ e têm vindo a demonstrar algum progresso na permeação cutânea ${ }^{[3-4]}$. A pele, o maior e mais pesado orgão, é responsável pela barreira que possibilita a entrada de fármacos no organismo. A camada mais externa da pele é o estrato córneo. Existem duas vias de permeação através do SC: via transepidérmica ou através dos poros. A via dos poros inclui os anexos da pele ${ }^{[5]}$. As unidades pilosebáceas são compostas por folículos pilosos e glândulas sebáceas ${ }^{[6]}$. Um dos problemas geralmente associado às formulações tópicas de fármacos é a sua fraca permeação, além dos problemas de solubilização e até mesmo de estabilidade. Com as nanopartículas poliméricas é esperado um aumento na estabilidade do fármaco, um aumento do tempo de residência da formulação e, consequentemente, um aumento da probabilidade da absorção do fármaco. Foi descrito em estudos anteriores ${ }^{[7]}$ que a permeação de partículas através da pele estava associada aos poros através dos folículos pilosos.

Neste estudo foi utilizado o ácido azelaico como fármaco modelo. $\mathrm{O}$ AzA possui propriedades bacteriostáticas, anti-inflamatórias, antiqueratinazantes e é utilizado no tratamento do acne ${ }^{[8,9]}$. Até ao momento, este fármaco não apresentou resistência bacteriana após tratamentos longos e demonstrou uma tolerabilidade favoráve ${ }^{[8,10]}$. Porém, a este fármaco está normalmente associada a necessidade de aplicações diárias e alguns problemas relacionados com a sua solubilidade ${ }^{[1]}$. O objectivo deste estudo centrou-se no desenvolvimento e caracterização de nanopartículas de PLGA contendo ácido azelaico. O PLGA (copolímero do ácido poli(DL-láctico-co-glicólico), um polímero biodegradável, tem sido vastamente utilizado em formulações de libertação controlada e tem demonstrado um perfil de degradação bastante favorável ${ }^{[12]}$. Neste estudo foram avaliados vários parâmetros tais como tamanho da partícula, potencial zeta e eficiência de encapsulação. 


\section{Materials and Methods}

\section{Chemicals}

Azelaic Acid (AzA) was obtained from SIGMAALDRICH $^{\mathrm{TM}}$ (Germany). PURASORB ${ }^{\circledR}$ PLDG 5002PLGA (ratio L/G\% 50:50 and MW 45.000-75.000 Da) was obtained from PURAC (Netherlands). Pluronic ${ }^{\circledR}$ F68 (POLX) was obtained from SIGMA-ALDRICH ${ }^{\mathrm{TM}}$ (USA) and Carbopol 940 was obtained from BF GOODRICH (Germany). All other reagents were of reagent grade.

\section{Nanoparticle preparation}

\section{AzA-loaded PLGA nanoparticles}

PLGA nanoparticles containing AzA were prepared by modified-spontaneous emulsification / solvent diffusion (m-SESD) method. A quasi-emulsification was prepared by dissolving $50 \mathrm{mg}$ of AzA and $100 \mathrm{mg}$ of PLGA in $5 \mathrm{~mL}$ of a solvent mixture of acetone:ethanol $(8: 2)$. The organic solution was added drop-by-drop with constant stirring (400 rpm) to aqueous solution with $0.1 \%$ of POLX at room temperature. The nanoparticle suspension was formed immediately as the aqueous phase turned milky. After the last drop, the organic solution was removed by rotary evaporation at $35-42^{\circ} \mathrm{C}$ for $10-15$ min until a nanoparticle precipitation was evident.

\section{Extraction Method}

To recover nanoparticles, the colloidal suspension was centrifuged at $40000 \mathrm{x}$ g for $40 \mathrm{~min}$ using a High Speed Table Top Centrifuge (Z36 HK, Hermle Labortechnik, Germany).

\section{Nanoparticle characterization}

\section{Particle size and zeta potential measurements}

The particle size and zeta potential of empty and AzAloaded PLGA nanoparticles (Figure 1) were measured by photon correlation spectroscopy and electrophoretic mobility with a Delsa ${ }^{\mathrm{TM}}$ NanoC

\section{Materiais e Métodos}

\section{Reagentes}

Ácido azelaico (AzA) foi obtido à SIGMAALDRICH $^{\mathrm{TM}}$ (Alemanha). O polímero PURASORB ${ }^{\circledR}$ PLDG 5002-PLGA (razão L/G\% 50:50 e PM 45000$75000 \mathrm{Da})$ foi adquirido à PURAC (Holanda). Pluronic $^{\circledR}$ F68 (POLX) foi adquirido à SIGMA$\mathrm{ALDRICH}^{\mathrm{TM}}$ (EUA) e Carbopol 940 à BF GOODRICH (Alemanha). Todos os restantes reagentes foram de grau analítico.

\section{Preparação das nanopartículas}

\section{Nanopartículas de PLGA contendo AzA}

As nanopartículas contendo AzA foram preparadas através do método modificado de emulsificação espontânea/difusão do solvente (m-SESD). Foi preparada uma emulsão através da dissolução de 50 mg de AzA e 100 mg de PLGA em 5 mL de uma mistura de acetona: etanol (8:2). A solução orgânica foi adicionada gota-a-gota, a uma velocidade de agitação constante (400 rpm), a uma solução aquosa de POLX a $0,1 \%$, à temperatura ambiente. A suspensão das nanopartículas formou-se instantaneamente e a fase aquosa tornou-se leitosa. Após a última gota, a solução orgânica foi removida recorrendo a um evaporador rotativo a $35-42^{\circ} \mathrm{C}$ durante 10 a 15 min até precipitação das nanopartículas.

\section{Método de recuperação}

Para recuperar as nanopartículas, a suspensão coloidal foi centrifugada a $40000 \mathrm{x}$ g durante $40 \mathrm{~min}$ recorrendo a uma centrífuga High Speed Table Top Centrifuge (Z36 HK, Hermle Labortechnik, Alemanha).

\section{Caracterização de nanopartículas}

\section{Avaliação do tamanho da partícula e potencial zeta}

A determinação do tamanho e o potencial zeta das nanopartículas de PLGA com e sem fármaco (Figura 1) foi feita através de espectroscopia de fotões e mobilidade electroforética, respectivamente, 
(Beckman Coulter, UK), respectively. Triplicate measurements were made before centrifugation. The Polydispersity index (P.I.) was also evaluated. recorrendo a um Delsa ${ }^{\mathrm{TM}}$ NanoC (Beckman Coulter, Reino Unido). As determinações foram realizadas antes do processo de recuperação e em triplicado. O índice de polidispersividade (I.P.) foi igualmente avaliado.

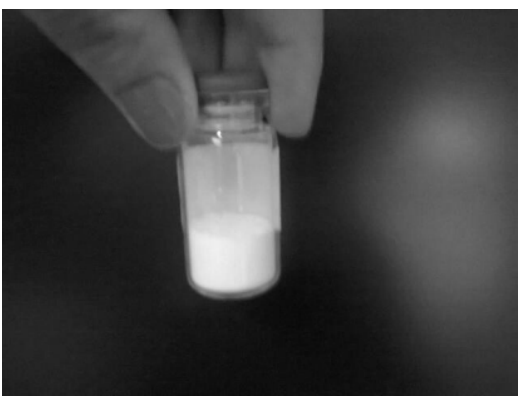

Figure 1 - AzA-loaded PLGA nanoparticles view.

Figura 1 - Aspecto das nanopartículas de PLGA contendo AzA.

\section{Encapsulation efficiency}

The encapsulation efficiency was determined after the supernatant centrifugation. Three batches were analyzed for free AzA by HPLC using the preservative benzoic acid as an internal standard (measured in triplicate) using a previously validated method $^{[13]}$.

\section{Production of a hydrophilic gel}

\section{Gelformulation}

The hydrophilic gel was prepared by dissolving 500 $\mathrm{mg}$ of Carbopol $940(1.5 \%, \mathrm{w} / \mathrm{w})$ in $100 \mathrm{~mL}$ of water. The solution was left stirring at room temperature to obtain an homogeneous solution. An aqueous solution of $\mathrm{NaOH}$ was carefully added, drop-by-drop, until gel consistency was observed.

\section{PLGA nanoparticle incorporation}

PLGA nanoparticles were incorporated into the Carbopol gel (11\%, w/w). A few drops of glycerol were added to the nanoparticles to facilitate incorporation. This formulation was stirred at room temperature at 13000 pulses per min for 15 min using ULTRATURRAX $^{\circledR}$ (Ika, Germany). The $\mathrm{pH}$ of the final gel was measured.

\section{Eficiência de encapsulação}

A eficiência de encapsulação foi determinada após centrifugação do sobrenadante. Foram analisados três lotes recorrendo a um método de HPLC, tendo como padrão interno o ácido benzóico (leituras feitas em triplicado), baseado em método previamente validado ${ }^{[13]}$.

\section{Produção de um gel hidrofilico}

\section{Formulação do gel}

O gel hidrofílico foi preparado dissolvendo $500 \mathrm{mg}$ de Carbopol $940(1,5 \%, \mathrm{~m} / \mathrm{m})$ em $100 \mathrm{~mL}$ de água. Esta solução foi agitada à temperatura ambiente até ficar homogénea. Posteriormente, foi adicionada uma solução aquosa de $\mathrm{NaOH}$ até à formação de um gel.

\section{Incorporação das nanopartículas de PLGA no gel}

As nanopartículas de PLGA foram incorporadas no gel de Carbopol $(11 \%, \mathrm{~m} / \mathrm{m})$. Foram adicionadas umas gotas de glicerina para facilitar a incorporação. Esta formulação foi ainda submetida a uma agitação com 13000 pulsos por min durante $15 \mathrm{~min}$ no ULTRATURRAX $^{\circledR}$ (Ika, Alemanha) e à temperatura ambiente. Foi avaliado o $\mathrm{pH}$ final do gel. 


\section{Results}

\section{Nanoparticle characterization}

Particle size and zeta potential

AzA-loaded PLGA and empty nanoparticle dimensons were $378.63 \mathrm{~nm}$ (P.I. 0.094) and $332.3 \mathrm{~nm}$ (P.I. 0.007), respectively. The surface zeta potential of the nanoparticles was $-7.82 \mathrm{mV}$ and $-14.92 \mathrm{mV}$ for drugloaded and empty nanoparticles respectively.

\section{Encapsulation efficiency}

Free AzA present in the supernatant was measured by HPLC and the value was $76.27 \pm 3.81 \%$. The method used was linear in the range of 100-500 ppm of azelaic acid. This method was linear and specific and, as seen in Figure 2, the peaks were completely baseline resolved.

\section{Resultados}

\section{Caracaterização das Nanoparticulas \\ Tamanho das partículas e potencial zeta}

Os tamanhos das nanopartículas de PLGA com e sem fármaco foram 378,63 nm (I.P. 0,094) e 332,3 nm (I.P. $0,007)$, respectivamente. O potencial zeta da superfície das nanopartículas foi de -7,82 $\mathrm{mV}$ e -14.92 mV para as nanopartículas com e sem fármaco, respectivamente

\section{Eficiência de encapsulação}

O AzA presente no sobrenadante foi quantificado por HPLC e o valor obtido foi $76,27 \pm 3,81 \%$. O método de quantificação do ácido azelaico por HPLC demonstrou ser linear entre 100-500 ppm. Este método é linear e específico e, como demonstra a Figura 2, os picos estão completamente resolvidos na linha de base.

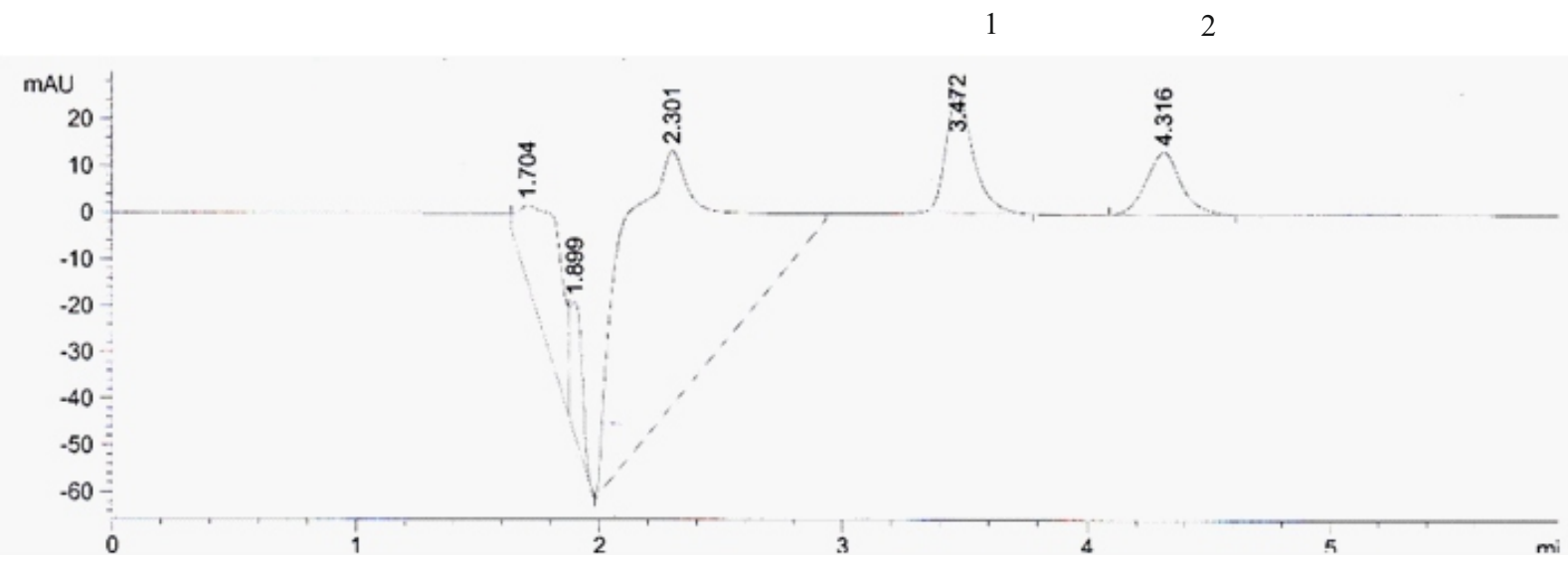

Figure 2 - HPLC chromatogram of AzA and benzoic acid. Peak 1 is AzA ( $350 \mathrm{ppm}$; Rt=3.472 min) and peak 2 is benzoic acid (100 ppm; Rt=4.316 min).

Figura 2 .- Cromatograma do AzA e ácido benzóico obtido através de HPLC. O pico 1 é AzA (350 ppm; Rt=3,472 min) e o pico 2 é o ácido benzóico (100 ppm; Rt=4,316 min).

\section{PLGA nanoparticle incorporation}

After PLGA nanoparticle incorporation at a concentration of $11 \%$, the gel became white with visible small agglomerates, and odourless. The gel was easily spread and washable as seen in Figure 3. The final gel $\mathrm{pH}$ was 3.52 .

\section{Incorporação das nanopartículas de PLGA}

Após incorporação das nanopartículas de PLGA numa concentração de $11 \%$, o gel tornou-se branco, com pequenos aglomerados e sem cheiro. $\mathrm{O}$ gel apresentava boa espalhibilidade e era de fácil remoção como demonstra a Figura 3. O pH final do gel foi de 3,52 . 


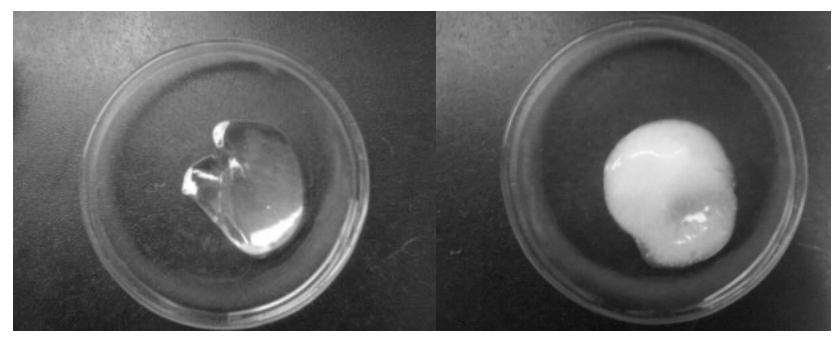

Higher concentration of nanoparticles into the gel did not demonstrate any technological advantage in terms of macroscopic characterization. For example, a $44 \%$ concentration was tested but the formulation became very stiff and pasty (Figure 4).

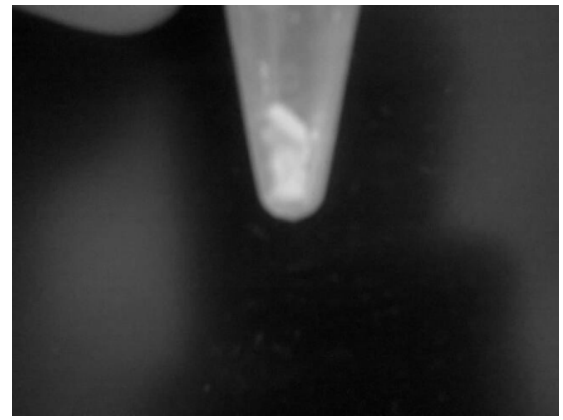

\section{Discussion}

Small, anionic and uniform particles were obtained by the m-SESD method. As shown in previous studies, these characteristics are crucial mainly because small, anionic nanoparticles have demonstrated better permeation in hair follicles ${ }^{[14]}$. In terms of skin permeation, Schneider et al. found that the optimum particle size for follicular permeation lies between 400-700 $\mathrm{nm}^{[15]}$, thus easily promoting transcutaneous permeation through pores. In terms of zeta, since skin has $+23 \mathrm{mV}$, negative particle charge, it may also increase the formulation residence time and increase the probability of drug absorption. The particle size and zeta potential revealed in this study also suggests that the AzA encapsulation occurred because a higher particle 108
Figure 3 - Gel before (A) and after (B) the incorporation of the nanoparticles.

Figura 3 - Gel antes (A) e após (B) incorporação das nanopartículas.

Concentrações mais elevadas de nanopartículas no gel não demonstraram nenhuma vantagem tecnológica em termos de caracterização macroscópica. Exemplo, foi testada uma concentração de $44 \%$ e a formulação tornou-se muito compacta e pastosa (Figura 4).

Figure 4 - Formulation with $44 \%$ of nanoparticles in gel.

Figura 4 - Formulação com $44 \%$ de nanopartículas no gel.

\section{Discussão}

Foram obtidas partículas pequenas, aniónicas e uniformes através do método m-SESD. Baseado em estudos anteriores ${ }^{[14]}$, é de facto importante que as nanopartículas apresentem um tamanho pequeno e um potencial zeta negativo uma vez que nestas condições poderão apresentar uma maior permeação nos folículos pilosos. Em termos de permeação cutânea, Schneider et al. demonstraram que o tamanho particular óptimo para a permeação folicular se encontra compreendido entre 400-700 nm ${ }^{[15]}$. A ser assim, o tamanho das nanopartículas obtido neste estudo poderá conduzir a uma maior permeação na pele através dos poros. Em termos do potencial zeta, considerando que a pele apresenta um potencial zeta de +23 
size and zeta potential were observed. Additionally, a significant part of AzA was efficiently encapsulated into PLGA nanoparticles. This value was confirmed in triplicate using an HPLC method. This HPLC method proved to be linear and two isolated peaks for AzA and benzoic acid were obtained.

\section{Conclusion}

AzA-loaded nanoparticles were developed in this study and these polymeric nanoparticles were shown to be an efficient encapsulation system for AzA. Future studies will include $\mathrm{pH}$ adjustment, evaluation of the permeation profile of the nanoparticle gel formulation by in vitro studies, efficacy against propionibacterium acnes, staphylococcus epidermis and staphylococcus aureus and excipient safety for human use.

\section{Conflict of interests}

The authors declare that there are no financial and personal relationships that could be viewed as presenting a potential conflict of interests.
$\mathrm{mV}$, uma partícula com carga negativa poderá aumentar ainda o tempo de residência da formulação e, consequentemente, a probabilidade de absorção do fármaco. O tamanho da partícula bem como o potencial zeta obtidos neste estudo sugerem ainda que a encapsulação do AzA ocorreu, uma vez que foram observados valores superiores em ambos os parâmetros após encapsulação. Além disso, observou-se uma boa eficiência de encapsulação de fármaco nas nanopartículas de PLGA. Este valor foi confirmado por HPLC em triplicado. O método de HPLC demonstrou ser linear e foram obtidos dois picos isolados de AzA e ácido benzóico.

\section{Conclusão}

Neste estudo foram desenvolvidas nanopartículas com AzA e estas nanopartículas poliméricas demonstraram ser um sistema de encapsulação eficiente do AzA. Estudos futuros incluirão ajustamento do $\mathrm{pH}$ do gel, uma avaliação da permeação das nanopartículas no gel através de estudos in vitro e estudos de eficácia contra propionibacterium acnes, staphylococcus epidermis e staphylococcus aureus e ensaios de segurança para uso em humanos.

\section{Conflito de Interesses}

Os autores declaram não existir qualquer relação pessoal ou financeira que possa ser entendida como representando um potencial conflito de interesses.

\section{References / Referências}

[1] Valizadeh H MG, Ehyaei R, Milani M, Azhdarzadeh M, Zakeri-Milani P, Lotfipour F. Antibacterial activity of clarithromycin loaded PLGA nanoparticles. Pharmazie 2012; 67: 63-8.

[2] Reis CP, Veiga FJ, Ribeiro AJ, Neufeld RJ and Damgé C. Nanoparticulate biopolymers deliver insulin orally eliciting pharmacological response. J. Pharm. Sci. 2008; 97: 5290305.

[3] Shah PP DP, Patel AR, Singh MS. Skin permeating nanogel for the cutaneous co-delivery of two anti-inflammatory drugs. Biomaterials 2012; 33: 1607-17.

[4] Tomoda K TH, Suzuki K, Inagi T, Terada H, Makino K. Enhanced transdermal delivery of indomethacin-loaded PLGA nanoparticles by iontophoresis. Colloids Surf B Biointerfaces 2011; 88: 706-10.

[5] Trommer H NR. Overcoming the stratum corneum: the modulation of skin penetration. Skin Pharmacol Physiol. 2006; 19: 106-21.

[6] Alsaad K Obaidat N GD. Skin adnexal neoplasms--part 1: an approach to tumours of the pilosebaceous unit. J Clin. Pathol. 2007; 60: 129-44.

[7] Buzea C PI, Robbie K. . Nanomaterials and nanoparticles: sources and toxicity. Biointerphases 2007; 2: 17-71.

[8] Elewski B DZ, Dréno B, Jansen T, Layton, Picardo M. . Rosacea - global diversity and optimized outcome: proposed international consensus from the Rosacea International Expert Group. . J Eur Academy of Derm Venereology 2011; 25: 188-200.

[9] Ramanathan S HA. Management of acne vulgaris. . Journal of pediatric health care : official publication of National Association of Pediatric Nurse Associates \& Practitioners 2003; 25: 43-51. [10] Tabari S NA, Hajian K, Moeinzadeh A. . Comparison of the effect of azelaic acid $20 \%$ and clindamycin $1 \%$ in the treatment of mild and moderate acne. Iranian J Derm. 2009; 106-10.

[11] Nan Li XW, Weibu Jia, Michelle C. Zhang, Fengping Tan, Jerry Zhan. Effect of ionization and vehicle on skin absorption and penetration of azelaic acid. Drug Development and Industrial Pharmacy 2011;

[12] Mainardes RM and Evangelista RC. PLGA nanoparticles containing praziquantel: effect of formulation variables on size distribution. Int. J. Pharm. 2005; 290: 137-44.

[13] Mansour A M IMM. Simultaneous determination of azelaic and benzoic acids in topical preparations by liquid chromatography. Chromatographia 2002; 55: 435-7. [14] Ogiso T YT, Iwaki M, Tanino T, Miyake Y. . Effect of positively and negatively charged liposomes on skin permeation of drugs. J. Drug Target. 2001; 9: 49-59. [15] Schneider M SF, Hansen S, Schaefer U. . Nanoparticles and their interactions with the dermal barrier. Dermato-endocrinology 2009; 1: 197-206. 